rearranging the position of the ports and bringing them closer to the tip of the femoral triangle. A 30 degree telescope helped in visualising the tip of the triangle better to remove the nodal tissue enbloc at completion of surgery.

Results Improved techniques led to easy identification of sartorius and standardization of the procedure.

Conclusion Issues and tips for improvement in surgical techniques especially in novel areas like robotic Inguino-femoral node dissection surgery are addressed.

Disclosures This surgical video was presented at IGCS Conference 2019.

\section{INTRA-TUMOURAL ELECTRO-CHEMOTHERAPY (ECT) WITH BLEOMYCIN FOR PALLIATION OF CUTANEOUS RECURRENCE IN GYNAECOLOGICAL MALIGNANCY}

${ }^{1}$ Stuart Rundle, ${ }^{1}$ Christine Ang, ${ }^{2}$ Tani Fasih, ${ }^{1}$ Porfyrios Korompelis, ${ }^{1}$ Nithya Ratnavelu. ${ }^{1}$ Northern Gynaecological Oncology Centre; Queen Elizabeth Hospital; ${ }^{2}$ Queen Elizabeth Hospital; Breast and General Surgery

\subsection{6/ijgc-2020-ESGO.183}

Introduction/Background ECT utilises pulsed electrical current to transiently increase cell membrane permeability to the cytotoxic agent, bleomycin. We present the use of ECT in patients with recurrent gynaecological malignancy previously treated with a combination of surgery or radiotherapy, experiencing symptoms from cutaneous lesions. This report presents evidence of the role for ECT in second and third line treatment. Methodology Between July 2017 and August 2019, 6 patients with cutaneous recurrence of gynaecological malignancy (5 vulvar SCC and 1 high grade serous ovarian cancer) were treated with intra-tumoural bleomycin (9000 iu or $15000 \mathrm{iu}$, dependent on tumour volume) and pulsed-probe electroporation. Response was assessed clinically in routine follow up or following self-referral with return of symptoms. Post procedure pain scores were collated as part the quality of life evaluation.

Results Median treated tumour diameter was $6 \mathrm{~cm}$ (range 2 $12 \mathrm{~cm}$ ). Pain scores peaked between day 2 and day 7 postprocedure. The median progression free interval was 3.6 months (range $0.8-6.7$ months).

Following ECT treatment 2 patients continued to receive supportive care. Two patients underwent repeat treatment with ECT and reported symptom improvement with each treatment. Due to further progression two patients underwent radical surgery and one patient received palliative chemotherapy.

Conclusion ECT should be considered for patients with symptomatic cutaneous recurrence of gynaecological malignancy who have previously had multi-modal treatment. It can achieve symptom control and reduce the need for radical surgery in this palliative setting.

Disclosures The Authors have no conflicts of interest to disclose.

\section{NEW VARIANT OF RECONSTRUCTIVE SURGERY FOR ADVANCED VULVAR CANCER TREATMENT}

\footnotetext{
${ }^{1}$ Olha Bubliieva, ${ }^{2}$ Yevgeniy Kostiuchenko, ${ }^{1}$ Valentyn Svintsitskiy, ${ }^{3}$ Igor Motuziuk, ${ }^{1}$ Nataly Tsip, 'Sergiy Nespryadko, ${ }^{1}$ Alena Samokhvalova, ${ }^{3}$ Oleg Sydorchuk. 'National Cancer Institute; ${ }^{2}$ National Cancer Institute; 0.0. Bogomolets National Medical University; ${ }^{3} 0.0$. Bogomolets National Medical University
}

10.1136/ijgc-2020-ESG0.184
Introduction/Background Surgical treatment of advanced vulvar malignant tumors usually requires immediate reconstruction. Large defects after pelvis, vagina, vulva, groin and perineum wide excision require closure with the usage of difficult reconstructive techniques. In this case the most suitable myocutaneous flap for reconstruction is rectus abdominis muscle flap, which provides the biggest volume of tissues to cover those large defects.

Methodology Woman 67 y.o. initially presented with the combined treatment of cervical cancer stage IIB. Within 5 month was diagnosed the lymphedema in the left lower extremity. Approximately 2 years she presented the vulvar tumor measured $15 \times 10 \mathrm{~cm}$. A biopsy was performed the lymphangiosarcoma.

Results In the National Cancer Institute of Ukraine we investigated a new variation of large defects reconstruction using rectus abdominis muscle flap. To collect a donor flap, we perform 3 arcuate incisions: one vertical by the medial line around the umbilicus, and two oblique incisions towards upper anterior iliac spine of one of the sides. In this way we use only one half of the abdomen, and in case of any complications with the flap or relapse of the disease we will have the second donor site for possible future reoperation. There is a narrow 'bridge' of tissues we leave between the excised donor and recipient sites. It is extremely important to preserve blood supply not only to the flap, but also to the 'bridge' to avoid complications. We perform a tunnel under the 'bridge' as small as possible to preserve all inferior epigastric vessels, but enough to transfer the flap and not to squeeze the pedicle. After the surgery we have a half-inverted Y-shaped scar on of the sides of the abdomen, an inverted triangular scar at the pelvis area, circumumbilical scar and a short vertical scar on the flap to imitate pudendal cleft with central structures of vulva (urethra and vaginal tube).

Conclusion We consider our variation of this type of surgery the most safe and efficient, with the opportunity of re-operation if needed. Advanced vulvar malignancies are quite rare, so we will keep working on development and enhancement of the technique to help these patients.

Disclosures Authors declare no disclosures.

\section{VULVAR RECONSTRUCTION IN PATIENTS WITH ADVANCED MALIGNANCIES}

'Olha Bubliieva, ${ }^{2}$ Yevgeniy Kostiuchenko, ${ }^{3}$ Igor Motuziuk, ${ }^{1}$ Valentyn Svintsitskiy, ${ }^{1}$ Sergiy Nespryadko, ${ }^{1}$ Nataly Tsip, ${ }^{3}$ Oleg Sydorchuk, ${ }^{1}$ Alena Samokhvalova. ${ }^{1}$ National Cancer Institute; ${ }^{2}$ National Cancer Institute; 0.0. Bogomolets National Medical University; ${ }^{3}$ O.O. Bogomolets National Medical University

\subsection{6/ijgc-2020-ESG0.185}

Introduction/Background Vulvar malignant tumors are the rarest oncological disease of women's reproductive system in Ukraine. In 2018 there were registered only 477 new cases of vulvar cancer among all Ukrainian women. It is approximately three cases per 100 thousand among female population. However there are less common histological types, such as leiomyosarcoma, melanoma and lymphangiosarcoma, which make up approximately $1 \%$ of all cases. The main goal in surgical treatment is to obtain gross surgical margins, which sometimes require the usage of reconstructive techniques.

Methodology 